\title{
Lesiones intracraneales originadas con pistola de bala cautiva
}

J.M. Santín-Amo; D. Castro-Bouzas; A. Arcos-Algaba; L. Díaz-Cabanas; R. Serramito-García; F.J. Bandín-Diéguez; J.M. Villa-Fernández y M. Gelabert-González

Servicio de Neurocirugía. Hospital Clínico Universitario de Santiago. Departamento de Cirugía. Universidad de Santiago de Compostela.

\section{Resumen}

Las pistolas de bala cautiva o clavija perforadora son empleadas para aturdir al animal como paso previo al sacrificio. Colocadas en la frente del mismo inducen una inmediata perdida de consciencia, logrando así una "humanización" del proceso. Su uso con fines suicidas es raro, casi exclusivo de personas que tienen acceso a las mismas dada su ocupación laboral, causando graves lesiones a nivel cerebral.

Analizamos tres casos que se presentaron en nuestro servicio. Uno de ellos fallece como consecuencia de las lesiones ocasionadas.

PALABRAS CLAVE: Pistola de bala cautiva. Pistola de clavija perforadora. Pistola de matarife. Tomografía computarizada. Traumatismo cráneo-cerebral.

Intracranial injury caused by captive bolt gun

\section{Summary}

Guns bullet or captive bolt is used as a weapon for stunning animals as a prelude to sacrifice. Placed in front of it induce an immediate loss of consciousness, thus achieving a "humanization" of process. It's use for suicide is rare, almost exclusively of people who have access to them because of their occupation, causing severe injuries to the brain. We analyse three cases presented in our service. One of them dies as a result of injuries.

KEY WORDS: Captive-bolt gun. Computed tomography. Cranio-cerebral trauma. Gunshot. Slaughterers' gun.

\section{Introducción}

Es ampliamente conocida la influencia del sexo, ocupación, lugar de residencia y estado psicológico de las perso-

Recibido: 25-01-10. Aceptado: 8-04-10 nas en la elección del modo de suicidio ${ }^{5,7}$. En este caso se trata fundamentalmente de varones de mediana edad, con antecedentes depresivos y que habitan en zonas rurales.

En los últimos años el uso de las pistolas de bala cautiva se ha extendido en España donde, a diferencia de USA o Inglaterra, no son precisos permisos especiales para su manejo, por lo que parte de la población, sobre todo en medios rurales, puede acceder a las mismas sin grandes dificultades.

\section{Casos clínicos}

Caso 1. Varón de 72 años de edad acude a urgencias tras intento autolítico presentando orificio de entrada a nivel frontal. Entre sus antecedentes destacan enolismo crónico y síndrome depresivo. Vive en una zona rural y ganadera. Presenta en la exploración una puntuación en la escala de Glasgow (GCS) de 14, sin apreciarse déficits asociados. Se practicó un estudio con tomografía computarizada (TAC) craneal que muestra trayecto de entrada a nivel frontal izquierdo con esquirlas óseas que llegan al asta frontal y hematoma intraparenquimatoso asociado (Figura 1).

Se realiza intervención quirúrgica urgente con lobectomía frontal izquierda, exéresis de los fragmentos óseos y reconstrucción de los planos dural, óseo y cutáneo. Al alta presenta, en la escala de resultados de Glasgow (GOS) una puntuación de 4 , sin focalidades e inicia tratamiento psiquiátrico.

Caso 2. Mujer de 45 años de edad que ingresa con carácter urgente presentando herida inciso-contusa en región temporal derecha tras intento autolítico. Entre los antecedentes destacan varios intentos de suicidio previos y esquizofrenia mal controlada. Vive en una zona rural y ganadera.

Al ingreso presenta una puntuación de 14 en la GCS, ptosis palpebral derecha con pupila midriática y arreactiva; hemiparesia izquierda $3 / 5$, y signo de Babinski izquierdo. En la TAC craneal se objetiva defecto óseo en región temporal derecha con esquirlas óseas a lo largo del trayecto y hematoma intraparenquimatoso asociado (Figura 2). 


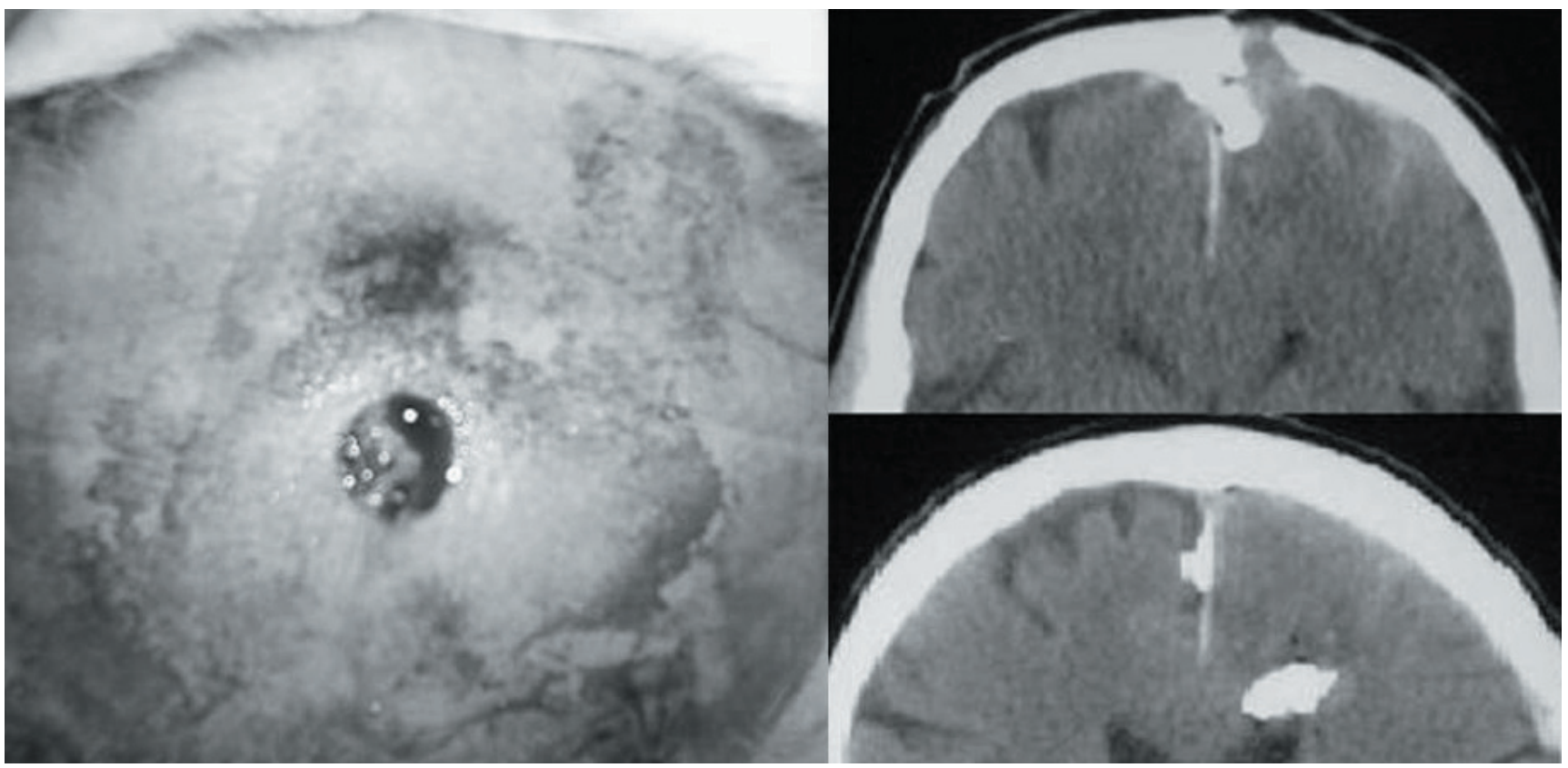

Figura 1. Orificio de entrada a nivel frontal. TAC al ingreso que muestra trayecto de entrada a nivel frontal izquierdo con esquirlas óseas que llegan al asta frontal y hematoma intraparenquimatoso asociado (caso 1).

Se realizó craneotomía fronto-temporal derecha con esquirlectomía, polectomía temporal y reconstrucción dural, ósea y cutánea. Al alta presenta, GOS de 4, mantiene la hemiparesia izquierda y la parálisis del III par derecho. Inicia tratamiento psicofarmacológico e ingresa en el hospital psiquiátrico

Caso 3. Varón de 66 años residente en zona rural que ingresa, tras intento autolítico, al ser encontrado inconsciente y con una herida en región frontal. Entre los ante- cedentes destacan el consumo excesivo de alcohol. A la exploración presenta una puntuación en la GCS de 4, y se encuentra sedado, intubado y relajado, con pupilas medias arreactivas. En el TAC craneal presenta un orificio de entrada a nivel de la línea media frontal, un hematoma que se extiende hasta cisterna interpeduncular y hemorragia subaracnoidea peritroncular. En todo el trayecto presenta múltiples esquirlas óseas y neumoencéfalo.

Dada su situación neurológica se descarta intervención

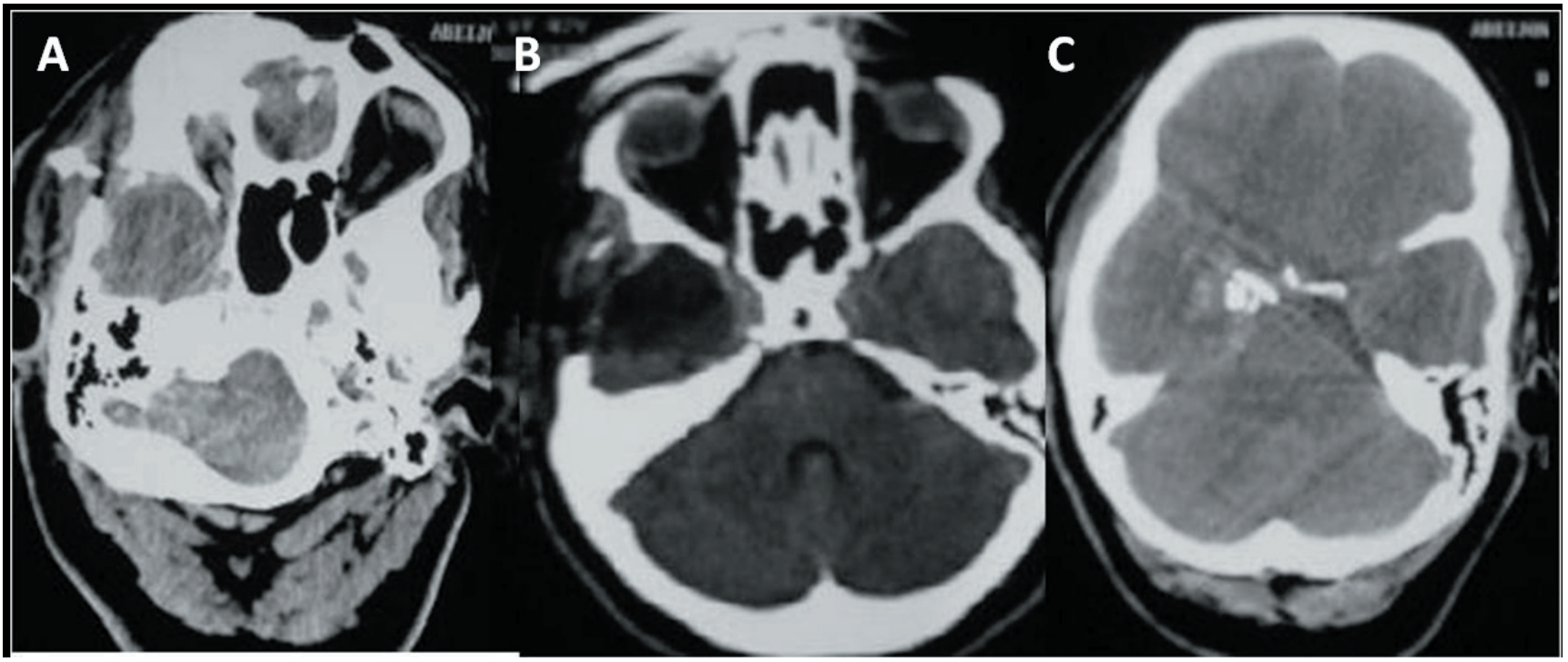

Figura 2. (A-C): TAC de llegada que muestra defecto óseo en región temporal derecha con esquirlas óseas a lo largo del trayecto. B: TAC al alta en el que es evidente la polectomía temporal derecha y la ausencia de tejidos extraños (caso 2). 


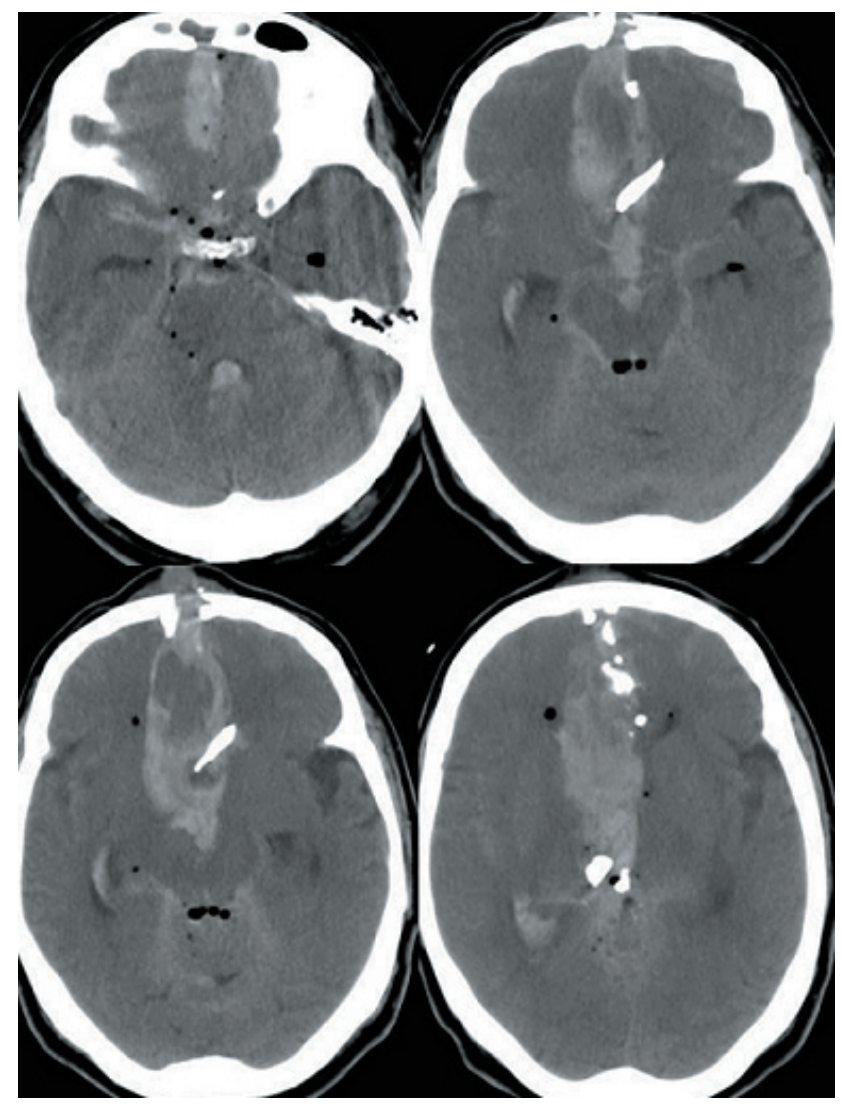

Figura 3. TAC inicial con orificio de entrada a nivel de la linea media frontal y un hematoma que se extiende hasta cisterna interpeduncular además de la hemorragia subaracnoidea peritroncular asociada (caso 3).

quirúrgica falleciendo el mismo día del ingreso.

\section{Discusión}

Los daños ocasionados con pistolas de bala cautiva o clavija perforadora son raros en nuestra área. En algunos países, entre ellos España, no se precisa licencia para su uso por lo que se trata de armas muy fáciles de conseguir por personas que se relacionan con el mundo ganadero como veterinarios, matarifes y los propios criadores ${ }^{2}$. Los daños accidentales y homicidios son menos frecuentes que las tentativas de suicidio ${ }^{1,14}$.

Consisten en un simple tubo cilíndrico con un perno metálico situado en su centro $(9-15 \mathrm{~cm}$ de largo y $1-1.5 \mathrm{~cm}$ de ancho) y dos orificios para el escape de gases. El perno es proyectado gracias a la descarga ocasionada por un cartucho de pólvora o aire comprimido y se retira por la acción de un muelle, alcanzando una velocidad máxima de $50 \mathrm{~m} / \mathrm{s}$ (Figura 4).

Los traumatismos penetrantes del cráneo se clasifican en función de la velocidad alcanzada por el proyectil, que

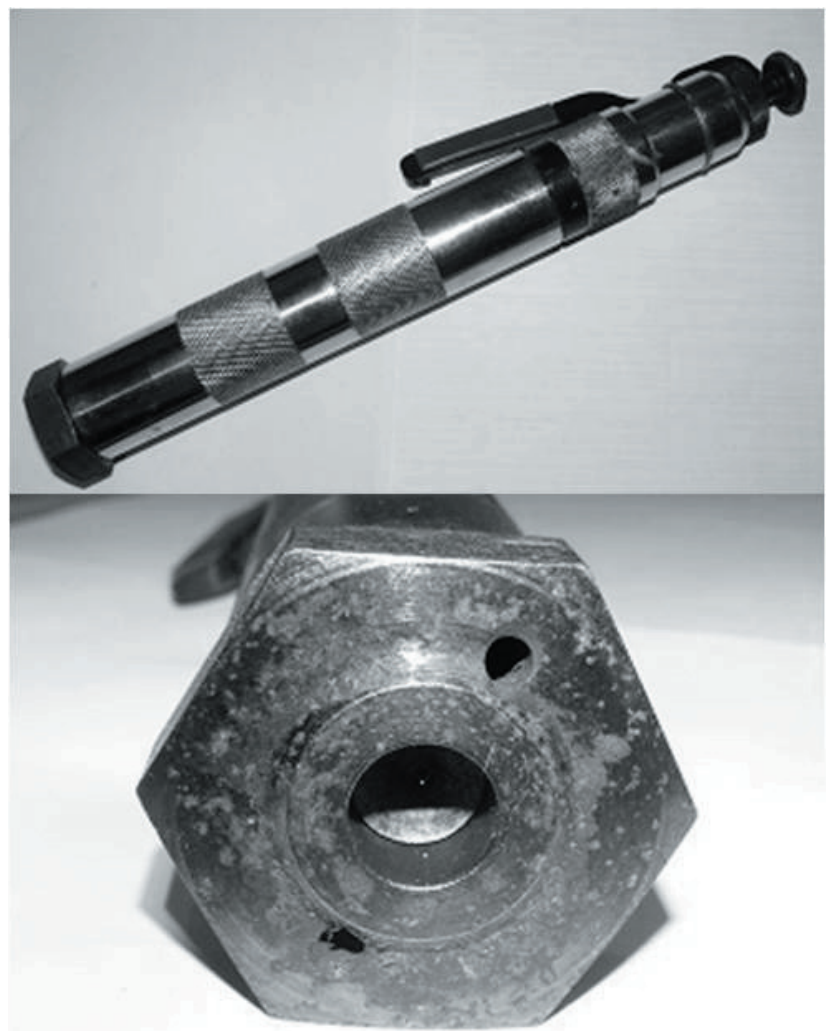

Figura 4. Pistola de clavija perforadora: tubo cilíndrico con un perno metálico situado en su centro $(9-15 \mathrm{~cm}$ de largo y $1-1.5 \mathrm{~cm}$ de ancho). En su parte frontal presentan dos orificios para el escape de gases.

es el factor lesional más importante. La energía cinética que este traslada al cráneo viene determinada por la fórmula matemática: $\mathrm{E}=1 / 2 \mathrm{M} \times(\mathrm{Ve}-\mathrm{Vs})$, donde $\mathrm{M}$ es la masa del proyectil y Ve y Vs las velocidades de entrada y salida del mismo $^{6}$. De acuerdo con la velocidad de entrada de los proyectiles, los traumatismos perforantes se clasifican en "baja velocidad" cuando ésta se encuentra entre 250-450 $\mathrm{m} / \mathrm{sg}$ (correspondería a armas cortas) y de "alta velocidad" cuando superan estas velocidades (correspondiendo a armas largas). Por lo tanto este tipo de lesiones formarían parte de un grupo especial denominado de "muy baja velocidad" en el que se incluyen también lesiones penetrantes por arma blanca o aquéllas ocasionadas por diferentes tipos de objetos metálicos 4,11 .

Las características de este tipo de armas hacen que las heridas sean diferentes de las ocasionadas por armas de fuego siendo la forma del orificio de entrada tan característica que sería reconocida fácilmente por personas con experiencia. Se trata de una lesión circular de la piel y hueso subyacente (tabla externa) con un diámetro similar al del perno percutor. Por el contrario el defecto interno es mucho mayor puesto que los fragmentos óseos desprendi- 
dos se comportan como proyectiles secundarios que se dispersan a lo largo del canal de disparo. Al final de este canal se encuentran siempre restos de piel, pelo y hueso ${ }^{5,12,13}$. El tamaño del mismo es siempre mayor al del perno debido al efecto de los proyectiles óseos ${ }^{9}$.

La profundidad de la herida y la dirección del perno percutor tienen relación con la afectación de importantes estructuras vasculares que causarían directamente la muerte ${ }^{1,5}$.

Dependiendo del ángulo de impacto del mismo, la forma de los residuos de pólvora que se proyectan a través de los orificios de salida es variable. Si el arma se coloca muy cercana al cuerpo la herida estará flanqueada por dos tatuajes de pólvora simétricos y circulares. Por el contrario al aumentar la distancia la intensidad de las manchas de pólvora se atenúa 5 .

De acuerdo al método propuesto por S. Pollak ${ }^{11}$ conociendo el ángulo formado por los conductos para la salida de gases con el eje central del perno y la distancia entre el centro de las manchas de pólvora y el centro de la herida podemos calcular el ángulo de entrada en el plano sagital.

En los estudios de imagen este tipo de lesiones presentan una serie de características típicas: punto de entrada con un importante defecto óseo en la tabla interna, un canal hiperdenso debido a la hemorragia, ocasionado por la entrada del perno y pequeños fragmentos óseos a lo largo del canal. En ocasiones se asocian edema cerebral y hemorragias intraventriculares o intraparenquimatosas. Al final de la herida siempre aparece un fragmento óseo de aproximadamente $1 \mathrm{~cm}$ de diámetro. Nunca se encuentran cuerpos extraños metálicos y no suele existir un orificio de salida lo que nos permite diferenciar este tipo de lesiones de las ocasionadas por armas de fuego ${ }^{8}$.

$\mathrm{Si}$ en todos los traumatismos perforantes es importante la limpieza adecuada de los tejidos y la extirpación de fragmentos de hueso y proyectil que puedan encontrarse sobre el lecho de la herida ${ }^{3}$ en estas lesiones debemos contar con el handicap de la contaminación primaria bien con flora de la piel o con gérmenes que pudiesen estar acantonados en el arma. Esta situación obliga a una minuciosa limpieza de las heridas para intentar reducir el alto riesgo de desarrollar meningitis y/o cerebritis que empeorarían sin lugar a dudas el pronóstico a largo plazo. Es por tanto primordial desbridar todo el tejido desvitalizado con el objetivo de eliminar todo cuerpo extraño, y así disminuir el riesgo de posteriores infecciones ${ }^{10,13}$. Como en todos los traumatismos penetrantes, debe realizarse una profilaxis antibiótica empírica que incluya un antibiótico de amplio espectro y metronidazol, así como valorar la necesidad de llevar a cabo profilaxis de crisis precoces con tratamiento anticomicial ${ }^{11,15}$.

Es también fundamental resolver todas aquellas lesiones que conlleven efecto masa, así como realizar una ade- cuada hemostasia y cierre durales, desaconsejándose el uso de sustitutos artificiales.

El pronóstico en estas lesiones penetrantes dependerá de varios factores, considerándose como de mal pronóstico la puntuación baja en la escala de Glasgow al ingreso, la elevación de la presión intracraneal, la presencia de hemorragia intraventricular, subaracnoidea o hematoma mayor de $15 \mathrm{~mL}$, efecto de masa y la existencia de desplazamiento de la línea media ${ }^{15}$. Es difícil lograr importantes daños si la pistola se coloca a una distancia superior a los $10 \mathrm{~cm}$ (vease paciente número 1) y ello podría explicar la baja incidencia de homicidios ${ }^{1}$. De acuerdo con la literatura la mortalidad es del $60-80 \%{ }^{9}$.

\section{Conclusiones}

El intento de suicidio con este tipo de armas es realmente raro en nuestro medio. La forma del orificio de entrada así como los patrones de los depósitos de pólvora y humo podrían ayudarnos a conocer tanto la dirección como el ángulo de impacto.

Este tipo de daño es habitualmente mortal debido a la afectación parenquimatosa, de grandes vasos o a la infección secundaria, alcanzando cifras que rondan el 65\% de mortalidad a pesar del tratamiento quirúrgico o antibiótico.

Frente al abundante número de publicaciones en el campo forense no existen muchos trabajos publicados a nivel neuroquirúrgico que describan el uso de pistolas de bala cautiva o clavija perforadora con fines suicidas. Es por tanto necesaria la aparición de mayores series que asienten las bases del tratamiento en este tipo de patologías.

\section{Bibliografía}

1. Betz, P., Pankratz, H., Penning, R., Eisenmenger, W.: Homicide with a captive bolt pistol. Am J Forensic Med Pathol 1993; 14: 54-57.

2. Bohnert, M., Schmidt, U., Perdekamp, M.G., Pollak, S.: Diagnosis of a captive-bolt injury in a skull extremely destroyed by fire. Forensc Sci Int 2002; 127: 192-197.

3. Dagi, T.: Emergency management of missile injuries to the brain: Resucitation, triage, and preoperative stabilization. Am J Emerg Med 1987; 5: 140-148.

4. Fernández-Melo, R., Morán, A.F., López-Flores, G., Bouza-Molina, W., García-Maeso, I., Benavides-Barbosas, J.: Trauma craneoencefálico perforante por arpón. Presentación de un caso y revisión de la literatura. Neurocirugía 2002; 13 : 397-400.

5. Gnjidić, Z., Kubat, M., Malenica, T., Sajko, T., Radić, I., Rumboldt, Z.: Epidemiological, forensic, clinical, and imaging characteristics of head injuries acquired in the suicide attempt with captive bolt gun. Acta Neurochir (Wien) 2002; 
144: 1271-1277.

6. Gregory, N.G., Lee, C.J., Widdicombe, J.P.: Depth of concussion in cattle shot by penetrating captive bolt. Meat Science 2007; 77: 499-503.

7. Hagemeier, L., Schyma, C., Madea, B.: Extended suicide using an atypical stud gun. Forensic Sci Int 2009; 189: en prensa.

8. Jagger, J., Dietz, P.: Death and injury by frearms: Who cares? JAMA 1986; 255: 3143-3144.

9. Marjan, D., Rumboldt, Z., Gotovac, N., Sajko, T.: CT Findings in patient with head injury from captive bolt gun. AJR Am J Roentgenol 2004; 182: 827-828.

10. Mosdal, C.: Cranio-cerebral injuries from slaughterer's gun. Acta Neurochir (Wien) 1985; 74: 31-34.

11. Okay, O., Daglioglu E., Ozdol, C., Uckun, O., Dalgic, A., Ergungor, F.: Orbitocerebral injury by a knife: case report. Neurocirugía 2009; 20: 467-469.

12. Pollak, S.: Morphology of injuries by "humane killer" (livestook stunner). Z Rechtsmed 1977; 26: 153-65.

13. Simic, M., Draskovic, M., Stojiljkovic, G.,Vukovic, R., Budimlija, Z.M.: The characteristics of head wounds
2010; 21: 491-495

inflicted by "humane killer" (captive-bolt gun)-a 15-year study. J Forensic Sci 2007; 52: 1182-1185.

14. Viel, G., Schröder, K., Püschel, K., Braun, C.: Planned complex suicide by penetrating captive-bolt gunshot and hanging: case study and review of the literature. Forensic Sci Int 2009; 187: en prensa.

15. Zazpe, I., Vázquez, A., Beaumont, C., Bardón, A., Azcona, J., Gallo-Ruiz, A., Portillo, E. Heridas múltiples penetrantes intracraneales causadas por pistola de clavos: caso clínico. Neurocirugía 2006; 17: 544-549.

Santín-Amo, J.M.; Castro-Bouzas, D.; Arcos-Algaba, A.; Díaz-Cabanas, L.; Serramito-García, R.; Bandín-Diéguez, F.J.; Villa-Fernández, J.M.; Gelabert-González, M.: Lesiones intracraneales originadas con pistola de bala cautiva. Neurocirugía 2010; 21: 491-495.

Correspondencia: José María Santín Amo. Servicio de Neurocirugía. Hospital Clínico Universitario de Santiago. A Choupana s/n. 15706 Santiago de Compostela

E-mail: cochemelide@hotmail.com 